\title{
New insights into the nature of the peculiar star $\theta$ Carinae ${ }^{\star}$
}

\author{
S. Hubrig ${ }^{1}$, M. Briquet ${ }^{2 \star \star}$, T. Morel ${ }^{2,3}$, M. Schöller ${ }^{1}$, J. F. González ${ }^{4}$, and P. De Cat ${ }^{5}$ \\ 1 European Southern Observatory, Casilla 19001, Santiago 19, Chile \\ e-mail: shubrig@eso.org \\ 2 Instituut voor Sterrenkunde, Katholieke Universiteit Leuven, Celestijnenlaan 200 D, 3001 Leuven, Belgium \\ 3 Institut d'Astrophysique et de Géophysique, Université de Liège, Allée du 6 Août, Bât. B5c, 4000 Liège, Belgium \\ ${ }^{4}$ Complejo Astronómico El Leoncito, Casilla 467, 5400 San Juan, Argentina \\ Koninklijke Sterrenwacht van België, Ringlaan 3, 1180 Brussel, Belgium
}

Received 15 April 2008 / Accepted 17 June 2008

\section{ABSTRACT}

\begin{abstract}
Context. $\theta$ Carinae belongs to a group of peculiar early-type stars (OBN) with enhanced nitrogen and carbon deficiency. It is also known as a binary system, but it is not clear yet whether the chemical anomalies can be explained by mass transfer between the two components. On the basis of the previously reported spectral variability of a few metal lines it may be expected that $\theta$ Car possesses a weak magnetic field.

Aims. A study of the physical nature of this hot massive binary which is furthermore a well-known blue straggler lying 2 mag above the turnoff of the young open cluster IC 2602 is important to understand the origin of its strong chemical anomalies.

Methods. We acquired high resolution spectroscopic and low resolution spectropolarimetric observations to achieve the following goals: a) to improve the orbital parameters to allow a more in-depth discussion on the possibility of mass transfer in the binary system; b) to carry out a non-local thermodynamic equilibrium (NLTE) abundance analysis; and c) to search for the presence of a magnetic field.

Results. The study of the radial velocities using CORALIE spectra allowed us to significantly improve the orbital parameters. A comparative NLTE abundance analysis was undertaken for $\theta$ Car and two other early B-type stars with recently detected magnetic fields, $\tau$ Sco and $\xi^{1} \mathrm{CMa}$. The analysis revealed significantly different abundance patterns: a one-order-of-magnitude nitrogen overabundance and carbon depletion was found in $\theta$ Car, while the oxygen abundance is roughly solar. For the stars $\xi^{1} \mathrm{CMa}$ and $\tau$ Sco the carbon abundance is solar and, while an $\mathrm{N}$ excess is also detected, it is of much smaller amplitude (0.4-0.6 dex). Such an N overabundance is typical of the values already found for other slowly-rotating (magnetic) B-type dwarfs. For $\theta$ Car, we attribute instead the chemical peculiarities to a past episode of mass transfer between the two binary components. The results of the search for a magnetic field using FORS 1 at the VLT consisting of 26 measurements over a time span of $\sim 1.2 \mathrm{~h}$ are rather inconclusive: only few measurements have a significance level of $3 \sigma$. Although we detect a periodicity of the order of $\sim 8.8 \mathrm{~min}$ in the dataset involving the measurements on all hydrogen Balmer lines with the exception of the $\mathrm{H} \alpha$ and $\mathrm{H} \beta$ lines, these results have to be confirmed by additional time-resolved magnetic field observations.
\end{abstract}

Key words. stars: early-type - stars: fundamental parameters - stars: abundances - stars: atmospheres - stars: magnetic fields stars: binaries: spectroscopic

\section{Introduction}

It has long been assumed that massive stars do not have magnetic fields, as they lack the convective outer mantle prevalent in lower mass stars. However, indirect evidence is supporting that magnetic fields are indeed present in massive $\mathrm{O}$ and early B-type stars (e.g., Henrichs et al. 2005; Rauw et al. 2001; Cohen et al. 2003; Gagné et al. 2005). Yet, only very few direct magnetic field detections have been reported in O-type stars so far (Donati et al. 2006a; Wade et al. 2006; Hubrig et al. 2007). Among the hottest B-type stars, a magnetic field has been discovered in the B0.2V star $\tau$ Sco (Donati et al. 2006b) and in the B0.7IV star $\xi^{1} \mathrm{CMa}$, which is one of the hottest $\beta$ Cephei stars, with a rather

^ Based on observations collected with the CORALIE spectrograph attached to the Euler Telescope of the Geneva Observatory located at La Silla (Chile), at the European Southern Observatory, Paranal, Chile (ESO programmes 67.D-0239(A), 072.D-0377(A), 078.D-0080(A) and 278.D-5056(A)), and at the Complejo Astronómico El Leoncito (CASLEO), Argentina.

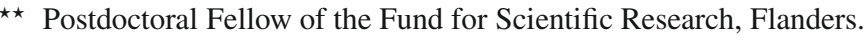

large longitudinal magnetic field of up to $300 \mathrm{G}$ (Hubrig et al. 2006).

Walborn (2006) listed the hot B0.2V star $\theta$ Carinae (HD 93030, HIP 52419, HR 4199; $m_{\mathrm{V}}=2.78$ ) among a few other massive stars with unexplained spectral peculiarities or variations for which a magnetic field could be expected. It has a peculiar, variable spectrum in both optical and UV. The spectral peculiarities in the blue-violet are an enhancement of nitrogen and deficiency of carbon, but also definite line-intensity variations as well as other line asymmetries (Walborn 1976, 1979).

The non-detection of a magnetic field was reported by Borra \& Landstreet (1979), who used a photoelectric Balmer-line magnetograph to measure circular polarization in the wings of the $\mathrm{H} \beta$ line. Although $\theta$ Car is a very bright target, easily observable with spectropolarimeters, no other magnetic field measurements have been reported so far in the literature, mainly due to the unavailability of instruments equipped with polarization analyzing optics on telescopes located in the southern hemisphere.

$\theta$ Car is an SB1 system with one of the shortest orbital periods known among massive stars $(P=2.2 \mathrm{~d}$; Lloyd et al. 1995). 
A discussion of the possibility of mass transfer in the binary system, which would be a natural explanation for the remarkable spectral peculiarities and for the singular location of this object in the H-R diagram of the 30 Myr old open cluster IC 2602, was presented by Walborn (1976). However, the spectral type of the companion remains unknown. Previously determined orbital elements were rather uncertain (Lloyd et al. 1995) and other periods of $\sim 1$ day and $\sim 25$ days have been suggested due to a systematic difference in the radial velocities from different observers.

Randich et al. (1995) reported in their ROSAT/PSPC study of the cluster IC 2602 that $\theta$ Car is the brightest X-ray object among the studied cluster members, with a soft X-ray luminosity amounting to up to $\log L_{\mathrm{X}}=30.99 \mathrm{erg} \mathrm{s}^{-1}$. Recently, Nazé \& Rauw (submitted), using XMM-Newton observations, showed that the X-ray flux of $\theta$ Car is slightly lower than the flux typically observed in $\mathrm{O}$ and early B-type stars and confirmed the unusual softness of the X-ray emission. Further, they noted that $\mathrm{X}$-ray lines appear narrow and unshifted, reminiscent of those of $\beta$ Cru and the magnetic star $\tau$ Sco (Donati et al. 2006b).

Below, we present the results of our new spectroscopic and spectropolarimetric study of this peculiar massive star and discuss possible origins of its anomalies.

\section{Orbital elements of the SB1 system}

The radial velocity (RV) of $\theta$ Car has been discovered to be variable almost a century ago (Wilson \& Sanford 1915). Although binarity is considered as the most plausible explanation for the observed short-period variations, the orbit was not well known prior to our study. The most recent orbit determination is given by Lloyd et al. (1995), who found a circular orbit with a period of $2.203020 \mathrm{~d}$ and an amplitude of $14.4 \mathrm{~km} \mathrm{~s}^{-1}$. It is based on all spectroscopic data that were available at that time. They found evidence for an additional period of about 25 days. However, they noted that both periods should be checked with supplementary data. To improve the orbit, 69 high-resolution $(R=47000)$ high signal-to-noise spectra $(S / N \sim 200$ at $4500 \AA)$ were taken with the CORALIE spectrograph attached to the $1.2 \mathrm{~m}$ Euler telescope (La Silla, Chile) from 2007 July 22 to 30. Exposure times were in the range 100-200 s. Thirteen additional spectra were taken between February and May 2008 at the CASLEO with the $2.1 \mathrm{~m}$ telescope and the REOSC spectrograph $(R=$ $13000)$ to improve the period determination. We also included in the radial velocity analysis a FEROS spectrum $(R=48000)$ obtained in February 2007 which was used for the chemical abundance analysis (see Sect. 3) and a FEROS spectrum obtained for the program 67.D-0239(A) in April 2001.

Radial velocities were measured by cross-correlations with a high- $S$ / $N$ spectrum of a star of similar spectral type (HD 37042, $\mathrm{B} 1 \mathrm{~V})$. The RV error of this template is about $0.5 \mathrm{~km} \mathrm{~s}^{-1}$, which we consider as the formal error of the zero point. The residuals of the fitted orbit indicate that the probable error in relative radial velocities is smaller (see below). During the orbit fitting we noted that CASLEO radial velocities show a systematic difference of about $0.9 \mathrm{~km} \mathrm{~s}^{-1}$ relative to CORALIE observations. To correct possible differences in the instrumental zero point, we measured the interstellar $\mathrm{Na}$ I doublet lines at $\lambda \lambda 5890,5896$. For these interstellar lines we obtained a mean radial velocity of $8.39 \pm 0.01 \mathrm{~km} \mathrm{~s}^{-1}$ for CORALIE spectra and $9.42 \pm 0.37 \mathrm{~km} \mathrm{~s}^{-1}$ for CASLEO spectra. Consequently, we applied a correction of $-1.03 \mathrm{~km} \mathrm{~s}^{-1}$ to the measured CASLEO velocities.

A Keplerian orbit was fitted by least-squares to the measured RVs listed in Table 1. Different weights have been assigned to the CORALIE and CASLEO observations due to their unequal
Table 1. Individual radial velocity measurements. Observations between MJD 54303 and MJD 54313 were taken with CORALIE, the CASLEO spectra between MJD 54520 and MJD 54581, while MJD 54141 and 52027 correspond to FEROS spectra.

\begin{tabular}{|c|c|c|c|c|c|}
\hline MJD & Phase & $\begin{array}{c}\mathrm{RV} \\
\mathrm{km} \mathrm{s}^{-1}\end{array}$ & MJD & Phase & $\begin{array}{c}\mathrm{RV} \\
\mathrm{km} \mathrm{s}^{-1}\end{array}$ \\
\hline 52027.0699 & 0.0939 & 06.00 & 54311.0013 & 0.8879 & 35.49 \\
\hline 54141.2248 & 0.8176 & 37.70 & 54311.0051 & 0.8896 & 35.32 \\
\hline 54303.9597 & 0.6913 & 36.03 & 54311.0090 & 0.8914 & 34.84 \\
\hline 54303.9902 & 0.7052 & 36.51 & 54311.0129 & 0.8931 & 34.66 \\
\hline 54304.0025 & 0.7108 & 36.95 & 54311.0168 & 0.8949 & 34.54 \\
\hline 54305.9760 & 0.6066 & 29.34 & 206 & 0.8966 & 34.60 \\
\hline 54305.9832 & 0.6099 & 29.43 & & 0.8984 & 34.33 \\
\hline 54305.9967 & 0.6160 & 30.04 & 54311.0283 & 0.9001 & 34.17 \\
\hline 54306.0049 & 0.6198 & 30.42 & 54311.0323 & 0.9020 & 33.92 \\
\hline 54306.0165 & 0.6250 & 30.73 & 54311.0366 & 0.9039 & 33.93 \\
\hline 54306.0250 & 0.6289 & 31.28 & 54311.0409 & 0.9059 & 33.92 \\
\hline 54306.0342 & 0.6331 & 31.29 & 54311.0451 & 0.9078 & 33.35 \\
\hline 54306.0400 & 0.6357 & 31.65 & 54311.0493 & 0.9097 & 33.48 \\
\hline 54306.0463 & 0.6386 & 31.69 & 54311.0535 & 0.9116 & 33.28 \\
\hline 54307.9612 & 0.5078 & 20.10 & 4311.9615 & 0.3238 & 05.08 \\
\hline 54307.9652 & 0.5096 & 20.24 & 4311.9701 & 0.3277 & 05.38 \\
\hline 54307.9694 & 0.5115 & 20.31 & 54311.9737 & 0.3293 & 05.47 \\
\hline 54307.9801 & 0.5164 & 20.89 & 54311.9777 & 0.3311 & 05.63 \\
\hline 54307.9960 & 0.5236 & 21.46 & 54311.9843 & 0.3341 & 05.83 \\
\hline 54308.0073 & 0.5288 & 21.91 & 21 & 0.3377 & 05.87 \\
\hline 08.9867 & 0.9734 & 24.61 & & 0.3393 & 06.05 \\
\hline 54308.9907 & 0.9752 & 24.35 & 54312.0112 & 0.3463 & 06.59 \\
\hline 54308.9949 & 0.9771 & 24.24 & 54312.0199 & 0.3503 & 06.93 \\
\hline 54308.9991 & 0.9790 & 23.88 & 54312.0299 & 0.3548 & 07.21 \\
\hline 54309.0029 & 0.9807 & 23.46 & 54312.0335 & 0.3564 & 07.41 \\
\hline 54309.0069 & 0.9825 & 23.32 & 54312.0390 & 0.3589 & 07.86 \\
\hline 54309.0109 & 0.9843 & 22.71 & 54312.0425 & 0.3605 & 07.92 \\
\hline 54309.0147 & 0.9861 & 22.67 & 54312.0471 & 0.3626 & 07.90 \\
\hline 54309.0186 & 0.9878 & 22.43 & 54312.0506 & 0.3642 & 08.20 \\
\hline 54309.0223 & 0.9895 & 22.30 & 54520.2392 & 0.8717 & 37.63 \\
\hline 54309.0260 & 0.9912 & 21.83 & 54521.2403 & 0.3262 & 05.73 \\
\hline 54309.0297 & 0.9929 & 21.72 & 54522.2258 & 0.7736 & 36.88 \\
\hline 54310.9623 & 0.8702 & 36.78 & 54544.2359 & 0.7651 & 38.05 \\
\hline 54310.9662 & 0.8719 & 36.79 & 54545.2886 & 0.2429 & 02.74 \\
\hline 54310.9700 & 0.8737 & 36.79 & 54546.2425 & 0.6759 & 36.26 \\
\hline 54310.9745 & 0.8757 & 36.39 & 54576.1355 & 0.2459 & 01.58 \\
\hline 54310.9783 & 0.8774 & 36.18 & 54577.1151 & 0.6906 & 34.80 \\
\hline 54310.9821 & 0.8792 & 36.25 & 54577.1842 & 0.7220 & 37.22 \\
\hline 54310.9860 & 0.8809 & 36.03 & 54578.0030 & 0.0937 & 07.68 \\
\hline 54310.9899 & 0.8827 & 35.83 & 54578.1381 & 0.1550 & 03.42 \\
\hline 54310.9937 & 0.8844 & 35.91 & 54579.1201 & 0.6008 & 26.86 \\
\hline 54310.9974 & 0.8861 & 35.29 & 54580.1620 & 0.0738 & 11.24 \\
\hline
\end{tabular}

probable errors: $0.15 \mathrm{~km} \mathrm{~s}^{-1}$ and $1.2 \mathrm{~km} \mathrm{~s}^{-1}$, respectively. These values were estimated from the residuals of the fitting itself. We found an orbital period of $2.20292 \pm 0.00005 \mathrm{~d}$. Once all orbital parameters were determined, we included in the analysis radial velocities previously published by Walborn (1979), Walker \& Hill (1985), and Lloyd et al. (1995). These old radial velocities can be fitted well with our radial velocity curve modifying slightly the period to $P=2.20288 \mathrm{~d}$. Finally, we kept the period fixed and re-fitted the remaining orbital parameters with our radial velocities. The orbital solution is plotted in Fig. 1 and the orbital elements are presented in Table 2. All our observations, which span over one year, are consistent, within the errors, with a single orbit. The error in the center of mass velocity is just the formal error of the fit, but the absolute error is larger. Besides, different lines in $\theta$ Car could possibly have slightly different velocities due to low-level spectral variability detected by 


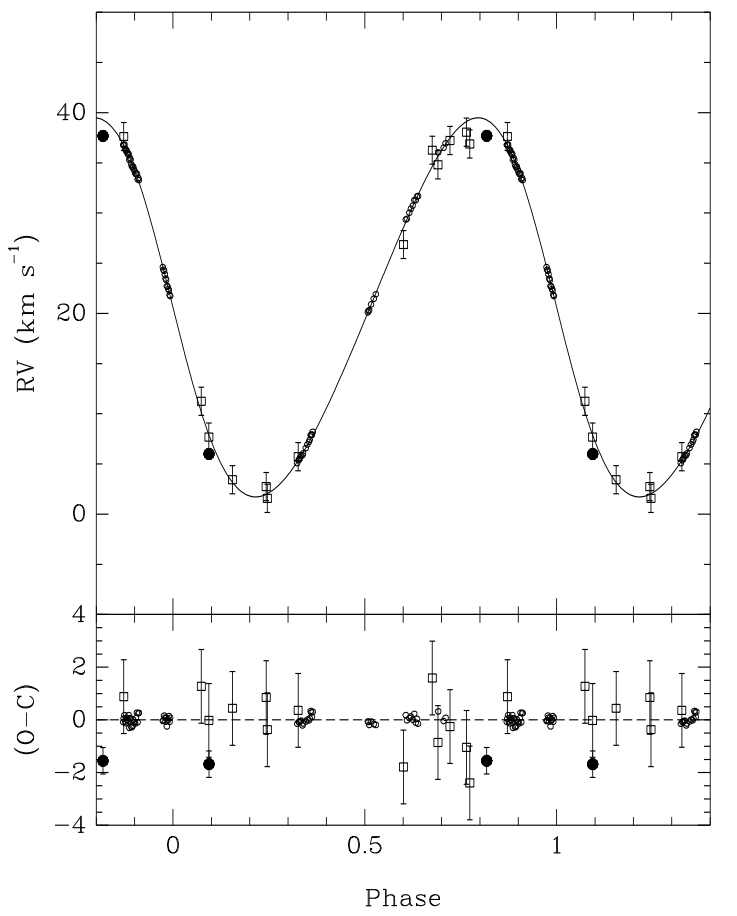

Fig. 1. Radial velocity curve of $\theta$ Car. Small open circles are observations from CORALIE, open squares from CASLEO, and the filled circles are from FEROS. The solid line corresponds to the adopted orbital solution. Residuals $(\mathrm{O}-\mathrm{C})$ are shown in the lower panel.

Table 2. Orbital elements for $\theta$ Car. $P$ is the orbital period, $v_{\gamma}$ is the system velocity, $K_{1}$ the semi-amplitude of the radial velocity curve of component $1, e$ is the eccentricity, $\omega_{1}$ the periastron longitude, $a_{1}$ the semi-major axis, $i$ the orbital inclination, $t$ (conj) the time of the primary conjunction, and $t$ (per) the time of the periastron passage.

\begin{tabular}{ll}
\hline \hline$P$ & $2.20288 \pm 0.00001 \mathrm{~d}$ \\
$v_{\gamma}$ & $20.18 \pm 0.04 \mathrm{~km} \mathrm{~s}^{-1}$ \\
$K_{1}$ & $18.93 \pm 0.05 \mathrm{~km} \mathrm{~s}^{-1}$ \\
$e$ & $0.129 \pm 0.002$ \\
$\omega_{1}$ & $81.8 \pm 1.7^{\circ}$ \\
$a_{1} \sin i$ & $0.00395 \pm 0.00014 \mathrm{AU}$ \\
$t$ (conj) & MJD 54 302.4367 \pm 0.0012 \\
$t$ (per) & MJD 54 302.3984 \pm 0.0092 \\
\hline
\end{tabular}

Walborn (1976). Thus it is not easy to decide which is the correct center-of-mass velocity.

The determined orbital period is in good agreement with the period found by Lloyd et al. (1995). However, we find a significant eccentricity $(e=0.13)$ and a higher amplitude $\left(K_{1}=\right.$ $18.9 \mathrm{~km} \mathrm{~s}^{-1}$ ). Furthermore, we do not find any definite evidence for the presence of secular changes in the orbital parameters or additional velocity variations, to a level of about $1 \mathrm{~km} \mathrm{~s}^{-1}$.

In Fig. 2 we present the older velocity measurements from the literature compared with our adopted orbit. Arbitrary velocity shifts have been applied to all three data samples as indicated in the figure caption. It is possible that the origin of these systematic differences is related to the use of spectral lines of different chemical elements and in different spectral regions for the measurements of radial velocities, although a long period variability cannot be completely discarded.

Due to the considerable number of CORALIE observations and the weights assigned, the orbital parameters are mainly determined by the CORALIE data and are representative of the orbit at the epoch of CORALIE observations, while the FEROS

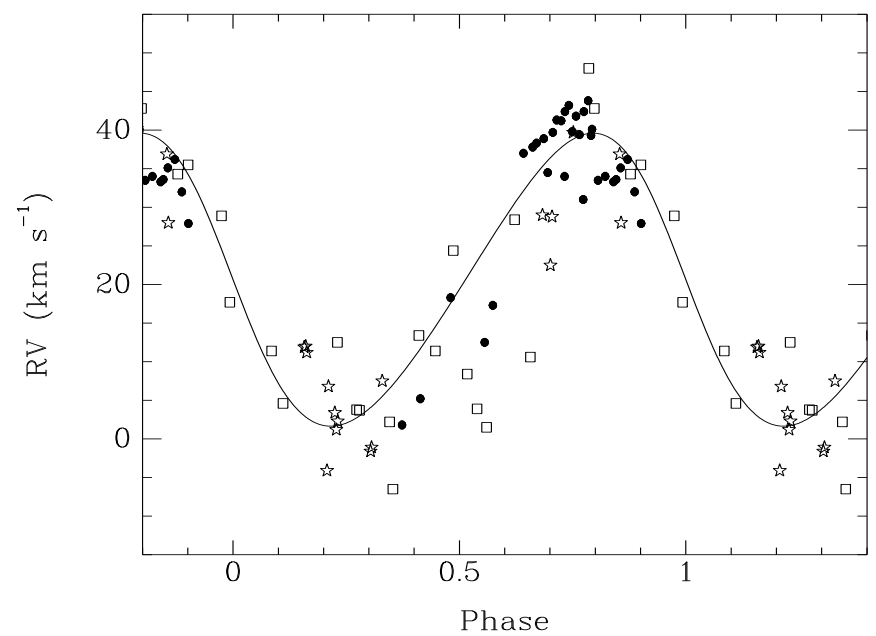

Fig. 2. Comparison of old radial velocity measurements with our calculated RV curve. Open squares: Walborn (1979) shifted by $-10 \mathrm{~km} \mathrm{~s}^{-1}$, open stars: Walker \& Hill (1985) shifted by $+10 \mathrm{~km} \mathrm{~s}^{-1}$, filled circles: Lloyd et al. (1995) shifted by $+15 \mathrm{~km} \mathrm{~s}^{-1}$.

and CASLEO spectra mainly contribute to a more precise determination of the orbital period. The sampling of our CORALIE data, however, is not adequate to allow us to detect a period as long as 25 days, which was mentioned by Lloyd et al. (1995).

The secondary component is not visible in our CORALIE spectra. In order to search for the secondary spectrum we proceeded in following way: first, each observed spectrum was shifted in wavelength by the amount corresponding to the primary velocity to allow the calculation of the mean spectrum of the primary component. This mean spectrum was subtracted from each observed spectrum. The residual spectra are expected to be low- $S / N$ spectra of the secondary star. To combine them it is necessary to know the secondary radial velocities, which are not a priori known. On the other hand, the radial velocity curve of the secondary can be calculated from the primary curve if a value for the mass-ratio is adopted. For a grid of mass-ratios in the range $0.03-0.60$ we calculated the corresponding velocity of the secondary star for the time of each observation, and applied corresponding shifts to the residual spectra. The average secondary spectrum was calculated for each assumed massratio. Finally we cross-correlated these spectra against templates in the range of spectral types A7-F9, which correspond to the temperatures expected for main-sequence stars of about 1-2 solar masses (see Sect. 5). For all considered mass-ratio values we find no evidence for the presence of a cross-correlation-peak corresponding to the secondary star. From tests with synthetic spectra with noise we estimate that a slowly rotating F-type star can be detected by the cross correlation even for a $S / N$-ratio as low as 1.3. Since the mean spectrum is expected to have a $S / N$ ratio of about 1400 with respect to the combined continuum, we conclude that, if the secondary star is an F-type star with moderate rotation, then the secondary spectrum contributes less than $0.1 \%$ to the total flux.

\section{Abundance analysis and spectral variability}

NLTE abundances of helium and several metals were calculated using the latest versions of the line formation codes DETAIL/SURFACE and plane-parallel, fully line-blanketed model atmospheres (Kurucz 1993). Note that ignoring the low luminosity companion in $\theta$ Car has no bearing on our abundance 
Table 3. Physical parameters of the programme stars (values for $\xi^{1} \mathrm{CMa}$ from Morel et al. 2006).

\begin{tabular}{lccc}
\hline \hline & $\xi^{1}$ CMa & $\tau$ Sco & $\theta$ Car \\
\hline Spectral type & B0.7 IV & B0.2 V & B0.2 V \\
$T_{\text {eff }}(\mathrm{K})$ & $27500 \pm 1000$ & $31500 \pm 1000$ & $31000 \pm 1000$ \\
$\log g\left(\mathrm{~cm} \mathrm{~s}^{-2}\right)$ & $3.75 \pm 0.15$ & $4.05 \pm 0.15$ & $4.20 \pm 0.20$ \\
$\xi\left(\mathrm{km} \mathrm{s}^{-1}\right)$ & $6 \pm 2$ & $2 \pm 2$ & $12 \pm 4$ \\
$v \sin i\left(\mathrm{~km} \mathrm{~s}^{-1}\right)$ & $10 \pm 2^{a}$ & $8 \pm 2$ & $113 \pm 8$ \\
\hline
\end{tabular}

${ }^{a}$ This value is an upper limit, as the spectral lines are significantly broadened by pulsations in this $\beta$ Cephei variable.

analysis. For comparison purposes, we also derived the abundances of the two magnetic early B-type dwarfs $\xi^{1} \mathrm{CMa}$ and $\tau$ Sco, following exactly the same procedures. Atmospheric models with a solar helium abundance were adopted, in accordance with the values found for all targets (see below). Our analysis of $\theta$ Car is based on a FEROS spectrum obtained on 2007 February 10 with the ESO/MPI 2.2 m telescope. This spectrum has a resolution similar to the CORALIE spectra used for the orbit determination in Sect. 2, but has a higher signal-to-noise ratio than the individual CORALIE spectra. For $\tau$ Sco, we used two CORALIE spectra obtained on 2007 March 18 and 2007 March 25 with the $1.2 \mathrm{~m}$ Euler Telescope at La Silla. In the case of the $\beta$ Cephei star $\xi^{1} \mathrm{CMa}$, we made use of the average of a large number of time-resolved CORALIE exposures primarily obtained for a study of its pulsational properties (see Saesen et al. 2006). The abundance results for this star are reported in Morel et al. (2006), where complete details on the methodology used to derive the elemental abundances can also be found.

A standard, iterative scheme is first used to derive the atmospheric parameters purely on spectroscopic grounds: $T_{\text {eff }}$ is determined from the Si III/IV ionization balance, $\log g$ from fitting the collisionally-broadened wings of the Balmer lines and the microturbulent velocity $\xi$ from requiring the abundances yielded by the $\mathrm{O}$ II features to be independent of the line strength. As a final step, the projected rotational velocity is inferred by comparing the profiles of a set of isolated metal lines with a grid of rotationally-broadened synthetic spectra. Support for our temperature scale comes from the good agreement between the abundances yielded by different ions for elements other than $\mathrm{Si}$. For $\tau$ Sco, both $\mathrm{C} \mathrm{II} / \mathrm{III}$ and $\mathrm{N}$ II/III yield mean abundance values differing by at most $0.11 \mathrm{dex}$, which is typical of the line-to-line scatter $\left(\sigma_{\text {int }}\right)$. The same conclusion holds for $\theta$ Car in the case of $\mathrm{N}$ III $\lambda 4634$, where the abundance is discrepant by only $\sim 2 \sigma_{\text {int }}$ from the mean value given by the $\mathrm{N}$ II lines. We infer a high microturbulent velocity in $\theta$ Car from the analysis of the $\mathrm{O}$ II lines compared to the usual values found for early B-type dwarfs ( $\xi=$ $12 \mathrm{~km} \mathrm{~s}^{-1}$ ). A similar result, within the errors, is obtained using other species (i.e. $\mathrm{N}$ and $\mathrm{Si}$ ).

The physical parameters for the three stars are provided in Table 3. An excellent agreement is found for $\tau$ Sco with other modern NLTE studies (e.g., Kilian 1994; Mokiem et al. 2005; Nieva \& Przybilla 2007; Repolust et al. 2005; Simón-Díaz et al. 2006). The same parameters within the errors are obtained for all studied stars when analyzing our data with the unified code FASTWIND (Puls et al. 2005) and (semi-)automatic line-profile fitting techniques (Lefever 2007). To our knowledge, the only previous abundance study dedicated to $\theta$ Car (Schönberner et al. 1988) led to a $T_{\text {eff }}$ value $1500 \mathrm{~K}$ higher than our estimate (as is also the case for $\tau$ Sco), but this can be naturally understood as a consequence of their use of only slightly line-blanketed atmospheric models (Kudritzki 1976).
Curve-of-growth techniques were used to determine the abundances using the equivalent widths of a set of unblended lines in the relevant temperature range. The line list was constructed after careful inspection of a B0 spectral atlas based on the extensive line list of Kurucz \& Bell $(1995)^{1}$. In the case of the fast rotator $\theta$ Car, the sharp-lined spectrum of $\tau$ Sco was used to further discard all diagnostic lines initially selected which could be significantly blended with nearby spectral features. No suitable carbon lines could be found owing to their extreme weakness (as already noted by Walborn 1976) and we followed in that case these following complementary approaches:

- although the C II $\lambda 4267$ doublet is unblended and reliably measurable, it is not appropriately modeled by our model atom and yields spuriously low abundances ${ }^{2}$. Nevertheless, the carbon abundance in $\theta$ Car can be roughly estimated through a differential analysis with respect to $\tau$ Sco in view of their similar physical parameters (Table 3) and the lack of evidence for a difference in metallicity (the Si and S abundances are uncertain, but agree within the errors; Table 4). We obtain an abundance in $\theta$ Car 0.98 dex lower than in $\tau$ Sco from the analysis of C II 24267 . Scaling to the mean $\mathrm{C}$ II abundance derived for the latter translates into an absolute abundance in $\theta$ Car: $\log \epsilon(\mathrm{C})=7.16 \mathrm{dex}$;

- alternatively, the $\mathrm{C}$ abundance can be inferred from a spectral synthesis of the blend formed by O II $\lambda 4185.4$ and C III 14186.9. We obtain an even lower best fit abundance: $\log \epsilon(\mathrm{C})=6.39$ dex (see Fig. 3).

Although we regard the carbon abundance yielded by C II $\lambda 4267$ as more reliable, and will only consider this value in the following, the C III $\lambda 4186.9$ analysis supports a very low carbon content and clearly rules out a solar value (as can be seen in Fig. 3). The abundances are given in Table 4 and are compared with the typical values found for nearby B-type dwarfs (e.g. Daflon \& Cunha 2004; Gummersbach et al. 1998; Kilian-Montenbruck et al. 1994). The quoted uncertainties are found by propagating the errors arising from the uncertainties on the atmospheric parameters and the line-to-line scatter.

A one-order-of-magnitude nitrogen overabundance and carbon depletion is found in $\theta$ Car, but the oxygen abundance is roughly solar. Schönberner et al. (1988) found $[\mathrm{N} / \mathrm{C}]=+1.7$ and $[\mathrm{N} / \mathrm{O}]=+0.3$, which agree, within the errors, with our values. Such abundance anomalies identify it as a member of the rare OBN class (Walborn 1976) and are consistent with its classification as a blue straggler (Schild \& Berthet 1986). Two main formation channels may account for the existence of this population in young open clusters.

On the one hand, they have been claimed to result from the quasi-chemically homogeneous evolution of single stars initially rotating at a significant fraction of breakup velocity (Maeder 1987). However, a strong $\mathrm{O}$ depletion and He enrichment may be expected, which is not the case (we do not confirm the moderate helium enhancement, $\mathrm{He} / \mathrm{H}=0.17$, reported by Schönberner et al. 1988). Very high rotation rates are also required in this scenario. In the recent models of Meynet \& Maeder (2003), even a $15 M_{\odot}$ star with a rotational velocity as high as $300 \mathrm{~km} \mathrm{~s}^{-1}$ on the Zero Age Main Sequence (ZAMS) follows a normal

\footnotetext{
1 Available online at:

http: //www. lsw . uni-heidelberg.de/cgi-bin/websynspec. cgi

${ }^{2}$ As is also the case for other NLTE line-formation codes (e.g. TLUSTY; Trundle et al. 2007). See, however, Nieva \& Przybilla (2007) for a recent solution to this long-standing problem.
} 
Table 4. Mean NLTE abundances (on the scale in which $\log \epsilon[\mathrm{H}]=12$ ) and total $1 \sigma$-uncertainties (values for $\xi^{1}$ CMa from Morel et al. 2006). The number of spectral lines used is given in brackets. A blank indicates that the abundance of a given element could not be determined. For comparison purposes, we provide in the last column the typical values found for OB dwarfs in the solar neighbourhood (Daflon \& Cunha 2004; Gummersbach et al. 1998; Kilian-Montenbruck et al. 1994). We define $[\mathrm{N} / \mathrm{C}]$ and $[\mathrm{N} / \mathrm{O}]$ as $\log [\epsilon(\mathrm{N}) / \epsilon(\mathrm{C})]$ and $\log [\epsilon(\mathrm{N}) / \epsilon(\mathrm{O})]$, respectively.

\begin{tabular}{lcccc}
\hline \hline & $\xi^{1} \mathrm{CMa}$ & $\tau$ Sco & $\theta$ Car & OB stars \\
\hline $\mathrm{He} / \mathrm{H}$ & $0.098 \pm 0.017(10)$ & $0.085 \pm 0.027(9)$ & $0.083 \pm 0.028(5)$ & $0.10^{a}$ \\
$\log \epsilon(\mathrm{C})$ & $8.18 \pm 0.12(9)$ & $8.19 \pm 0.14(15)$ & $7.16 \pm 0.46(1)^{b}$ & $\sim 8.2$ \\
$\log \epsilon(\mathrm{N})$ & $8.00 \pm 0.17(34)$ & $8.15 \pm 0.20(35)$ & $8.56 \pm 0.27(12)$ & $\sim 7.6$ \\
$\log \epsilon(\mathrm{O})$ & $8.59 \pm 0.17(34)$ & $8.62 \pm 0.20(42)$ & $8.38 \pm 0.22(7)$ & $\sim 8.5$ \\
$\log \epsilon(\mathrm{Mg})$ & $7.37 \pm 0.20(1)$ & $7.45 \pm 0.09(2)$ & & $\sim 7.4$ \\
$\log \epsilon(\mathrm{Al})$ & $6.16 \pm 0.22(4)$ & $6.31 \pm 0.29(3)$ & & $\sim 6.1$ \\
$\log \epsilon(\mathrm{Si})$ & $7.13 \pm 0.21(4)$ & $7.24 \pm 0.14(9)$ & $7.43 \pm 0.23(10)$ & $\sim 7.2$ \\
$\log \epsilon(\mathrm{S})$ & $6.99 \pm 0.16(2)$ & $7.18 \pm 0.28(3)$ & $7.32 \pm 0.33(1)$ & $\sim 7.2$ \\
$\log \epsilon(\mathrm{Fe})$ & $7.30 \pm 0.22(32)$ & $7.33 \pm 0.31(13)$ & & $\sim 7.3^{c}$ \\
\hline$[\mathrm{N} / \mathrm{C}]$ & $-0.18 \pm 0.21$ & $-0.04 \pm 0.25$ & $+1.40 \pm 0.53$ & $\sim-0.6$ \\
{$[\mathrm{~N} / \mathrm{O}]$} & $-0.59 \pm 0.25$ & $-0.47 \pm 0.29$ & $+0.18 \pm 0.35$ & $\sim-0.9$ \\
\hline
\end{tabular}

${ }^{a}$ From Lyubimkov et al. (2004); ${ }^{b}$ derived from a differential analysis of the C II $\lambda 4267$ doublet with respect to $\tau$ Sco (see text); ${ }^{c}$ mean NLTE abundance from Morel et al. (2006).

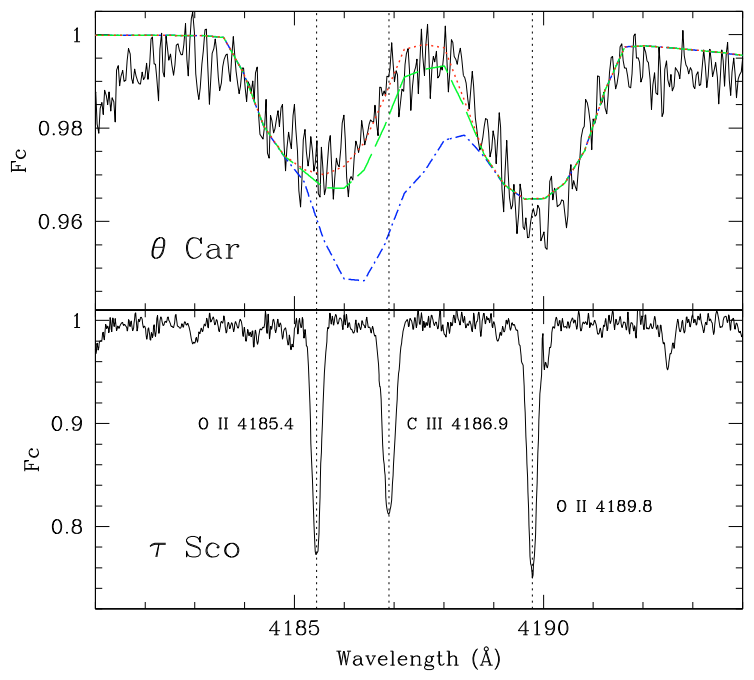

Fig. 3. Upper panel: spectral synthesis in $\theta$ Car of the blend formed by O II $\lambda 4185.4$ and C III $\lambda 4186.9$. Solid line: observed spectrum, dottedshort dashed line: synthetic spectrum with a carbon abundance typical of B-type dwarfs $(\log \epsilon[\mathrm{C}]=8.20$ dex; see Table 4), long-dashed line: synthetic spectrum with the carbon abundance yielded by the C II $\lambda 4267$ doublet $(\log \epsilon[C]=7.16$ dex $)$, dotted line: synthetic spectrum with the best fit carbon abundance $(\log \epsilon[\mathrm{C}]=6.39 \mathrm{dex})$. In the online version of this journal, the synthetic spectra are plotted as blue, green and red lines, respectively. A microturbulent velocity, $\xi=12 \mathrm{~km} \mathrm{~s}^{-1}$, and an oxygen abundance, $\log \epsilon(\mathrm{O})=8.38 \mathrm{dex}$, were assumed in all cases. All synthetic spectra have been convolved with a rotational broadening function with $v \sin i=113 \mathrm{~km} \mathrm{~s}^{-1}$ (see Tables 3 and 4). Lower panel: the same spectral region in $\tau$ Sco with the most prominent spectral lines identified. Note the different $y$ scale.

redward evolution. Furthermore, complete mixing on the main sequence is much more likely to occur in low-metallicity environments because of the small loss of angular momentum resulting from the much lower mass-loss rates (e.g. Woosley \& Heger 2006). On the other hand, there is strong observational evidence in dwarfs for a link between the OBN phenomenon and binarity (e.g. Levato et al. 1988). The high rotation rate and altered $\mathrm{CN}$ abundances would result in that case from the accretion during a past episode of mass transfer of incomplete CNOcycle processed material from the initially more massive component. In the framework of this binary scenario, the solar helium abundance would indicate that complete mixing of the mass gainer has not taken place during the accretion process (Vanbeveren \& de Loore 1994).

A different abundance pattern is found in the single stars $\xi^{1} \mathrm{CMa}$ and $\tau$ Sco. First, the carbon abundance is solar and, while an $\mathrm{N}$ excess is also detected, it is of much smaller amplitude $(0.4-0.6 \mathrm{dex})$. This $\mathrm{N}$ overabundance is typical of the values already found for other slowly-rotating (magnetic) B-type dwarfs and may require another physical explanation (Morel et al. 2008).

In our CORALIE spectra, the Si III absorption lines show weak variability over the orbital period (Fig. 4). The Si III line at $\lambda 4552.6$ displays an asymmetric profile at phases 0.71 and 0.98-0.99, while it is rather symmetric at phases $0.87-0.91$. The blend of the two Si III lines at $\lambda 4819.6 / \lambda 4819.7$ seems to have a sharper core (like a triangle) in phases $0.6-0.9$ and is more round or square shaped in the phase range $0.3-0.5$. The detected spectral variability could be caused by the presence of surface Si spots, by pulsations, or by the presence of the companion. Our spectral material is, however, insufficient to test the origin of this variability. We believe that also the He I lines are weakly variable, but a detailed study of the presence of He and $\mathrm{Si}$ spots on the surface of $\theta$ Car requires much higher spectral resolution and higher $S / N$ spectra. Interestingly, a similar lowlevel variability in He lines has been detected in the spectra of the SB1 system HD 191612 of spectral type Of?p (Nazé et al. 2007), for which a magnetic field has been detected by Donati et al. (2006a).

\section{Spectropolarimetric observations}

The only search for a mean longitudinal magnetic field in $\theta$ Car before our FORS 1 observations has been conducted by Borra \& Landstreet (1979) using a photoelectric Pockels cell polarimeter for the measurement of the circular polarization in the wings of the $\mathrm{H} \beta$ line. Measuring the mean longitudinal magnetic field is currently the standard method for searching magnetic fields in different types of stars. The mean longitudinal magnetic field is the average over the visible stellar hemisphere of the component of the magnetic field vector along the line of sight. Due to the sensitivity to aspect, it provides essential constraints for all models of geometry and gives a detailed structure of the magnetic fields of these stars. 

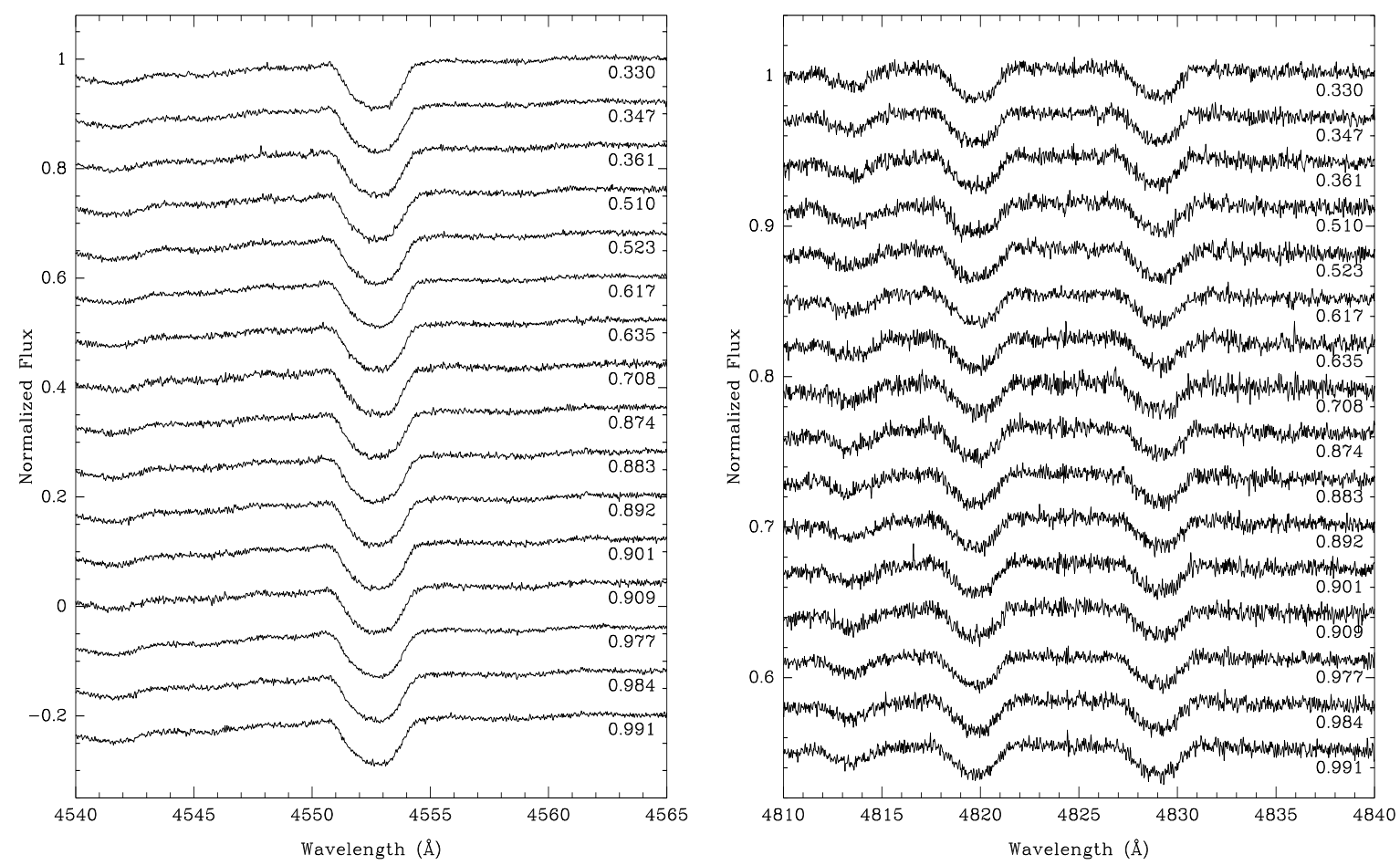

Fig. 4. Weak spectral line variability of Si III lines in $\theta$ Car. Left: Si III at $\lambda 4552.6$. Right: Si III at $\lambda 4819.6 / \lambda 4819.7$ and $\lambda 4829.0$. The normalized spectra are from 16 different orbital phases. Each spectrum is an average of three to five CORALIE spectra obtained close in time. The spectra are shifted vertically for display purposes.

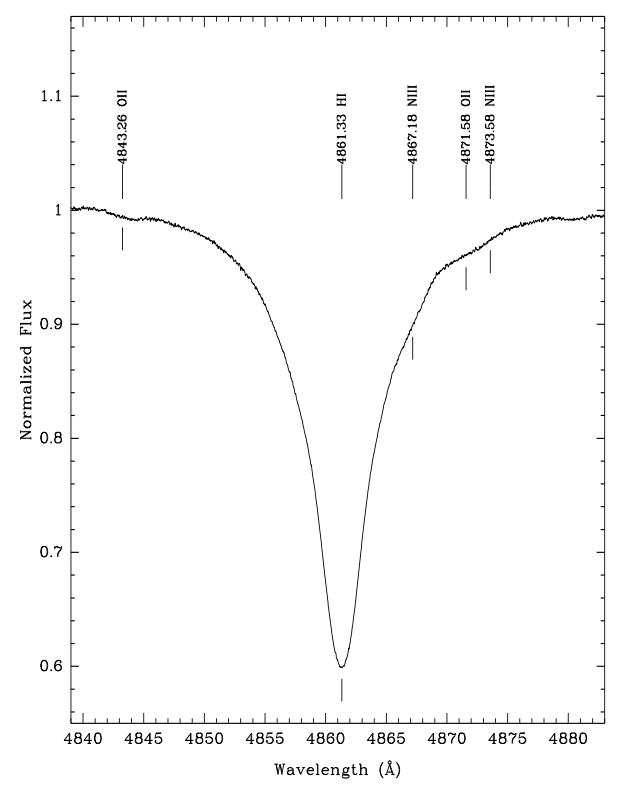

Fig. 5. The $\mathrm{H} \beta$ line for $\theta$ Car with $\mathrm{N}$ III blends in the red wing.

$\theta$ Car was observed by Borra \& Landstreet (1979) on four nights with exposure times between 40 and $64 \mathrm{~min}$, but no field was detected in spite of rather small standard deviations of the order of 50-65 G. A presence of variable emission in the $\mathrm{H} \beta$ line, which could possibly dilute the Zeeman polarization signal, was not reported by previous spectroscopic studies, hence the non-presence of a magnetic field seemed to be justified.

The careful inspection of our FEROS spectrum and CORALIE spectra of $\theta$ Car does not reveal any emission-like feature in the core of the $\mathrm{H} \beta$ line. However, we detect a clear blend contribution in the red wing of this line, mainly due to
$\mathrm{N}$ III lines (Fig. 5). As this element is found overabundant in $\theta$ Car by a one-order magnitude, the blend can in principle affect the previous magnetic field measurements which made use of circular polarization present in the wings of the $\mathrm{H} \beta$ line.

The first observations of $\theta$ Car with FORS 1 at the VLT in polarimetric mode using GRISM 600B were obtained in 2004 January in the framework of our survey of magnetic fields in Ap and Bp stars with accurate Hipparcos parallaxes (Hubrig et al. 2007). The observations consisted of four series of two exposures with the retarder waveplate oriented at two different angles $\left(\alpha=+45^{\circ}\right.$ and $\left.-45^{\circ}\right)$. During this run we used the GRISM 600B to cover the whole spectral region from $\mathrm{H} \beta$ to the Balmer jump. With the narrowest available slit width of 0.4 we could achieve a spectral resolving power of $R \approx 2000$. The highest signal-to-noise ratio at $\sim 4300 \AA$ was about 650 . Quite puzzling, the magnetic field measurements on the Stokes $V$ spectra obtained with an exposure time of $0.2 \mathrm{~s}$ showed a change of the field polarity after the first series (which took $2.5 \mathrm{~min}$ ) from a positive magnetic field to a negative magnetic field. The polarity change could, however, not obviously be explained by any instrumental effect since no change of polarity on short time scales was observed in the magnetic Ap star HD 99563, observed in the same night just after $\theta$ Car. To solve the puzzle, we reobserved this star in 2007 March at higher spectral resolution and at higher $S / N(S / N \sim 1200)$ with the GRISM 1200B in the spectral region from $3805 \AA$ to $4960 \AA$ at a spectral resolving power of $R \approx 4000$. To monitor the behaviour of the magnetic field over at least a part of the stellar surface we carried out a time series of exposures with short integration time over the time span of $\sim 1.2 \mathrm{~h}$. In all, we obtained 26 series of magnetic field measurements with exposure times of $0.8 \mathrm{~s}$ and $1.1 \mathrm{~s}$. An additional star with a well-defined strong longitudinal field, HD 96440, was selected as a standard star to check that the instrument was functioning properly. This star has a mean longitudinal magnetic field 
Table 5. Individual measurements of the mean longitudinal magnetic field for $\theta$ Car for the observations in 2004 and 2007.

\begin{tabular}{cr|r|r|r|r}
\hline \hline MJD & time & \multicolumn{1}{|c|}{$\begin{array}{c}\left\langle B_{z}\right\rangle \pm \sigma_{B} \\
{[\mathrm{G}]}\end{array}$} & \multicolumn{1}{|c}{$\begin{array}{c}\left\langle B_{z}\right\rangle \pm \sigma_{B} \\
{[\mathrm{G}]}\end{array}$} & \multicolumn{1}{|c}{$\left\langle B_{z}\right\rangle \pm \sigma_{B}$} & phase \\
& & \multicolumn{1}{c}{ set 1} & \multicolumn{1}{c}{ set 2} & \multicolumn{1}{c}{ set 3} & \\
\hline 53012.22798 & 0.0 & $247 \pm 130$ & $692 \pm 184$ & $322 \pm 211$ & \\
53012.22973 & 151.2 & $-136 \pm 131$ & $-267 \pm 186$ & $-584 \pm 216$ & \\
53012.23149 & 303.3 & $-219 \pm 125$ & $-238 \pm 178$ & $-342 \pm 206$ & \\
53012.23324 & 454.5 & $-122 \pm 142$ & $-176 \pm 205$ & $-242 \pm 223$ & \\
\hline 54181.00814 & 0.0 & $-46 \pm 52$ & $-223 \pm 92$ & $-47 \pm 112$ & 0.000 \\
54181.01007 & 166.1 & $49 \pm 62$ & $148 \pm 78$ & $113 \pm 98$ & 0.316 \\
54181.01214 & 345.3 & $-106 \pm 55$ & $-25 \pm 77$ & $18 \pm 96$ & 0.656 \\
54181.01421 & 524.1 & $-57 \pm 45$ & $69 \pm 83$ & $56 \pm 101$ & 0.996 \\
54181.01613 & 690.2 & $57 \pm 55$ & $26 \pm 90$ & $1 \pm 110$ & 0.311 \\
54181.01805 & 856.3 & $34 \pm 47$ & $-38 \pm 78$ & $-226 \pm 97$ & 0.627 \\
54181.01997 & 1022.2 & $53 \pm 47$ & $84 \pm 88$ & $100 \pm 109$ & 0.942 \\
54181.02190 & 1188.2 & $48 \pm 50$ & $136 \pm 82$ & $207 \pm 102$ & 0.258 \\
54181.02382 & 1354.2 & $-49 \pm 58$ & $-51 \pm 95$ & $-185 \pm 120$ & 0.573 \\
54181.02574 & 1520.3 & $266 \pm 52$ & $220 \pm 85$ & $244 \pm 108$ & 0.889 \\
54181.02766 & 1686.5 & $53 \pm 57$ & $85 \pm 93$ & $-37 \pm 118$ & 0.204 \\
54181.02959 & 1853.0 & $-81 \pm 60$ & $-239 \pm 100$ & $-305 \pm 124$ & 0.521 \\
54181.03153 & 2020.3 & $64 \pm 50$ & $32 \pm 82$ & $219 \pm 104$ & 0.839 \\
54181.03345 & 2186.6 & $26 \pm 51$ & $-3 \pm 84$ & $-5 \pm 105$ & 0.155 \\
54181.03537 & 2352.5 & $-131 \pm 72$ & $-245 \pm 92$ & $-459 \pm 118$ & 0.470 \\
54181.03730 & 2519.2 & $182 \pm 65$ & $176 \pm 105$ & $423 \pm 132$ & 0.786 \\
54181.03960 & 2717.6 & $81 \pm 45$ & $-77 \pm 75$ & $58 \pm 93$ & 0.164 \\
54181.04154 & 2885.4 & $-210 \pm 52$ & $-202 \pm 87$ & $-287 \pm 110$ & 0.482 \\
54181.04348 & 3053.4 & $37 \pm 50$ & $-92 \pm 80$ & $-86 \pm 103$ & 0.802 \\
54181.04542 & 3220.4 & $118 \pm 50$ & $45 \pm 84$ & $205 \pm 105$ & 0.119 \\
54181.04736 & 3388.5 & $-161 \pm 62$ & $98 \pm 102$ & $-180 \pm 129$ & 0.438 \\
54181.04931 & 3556.5 & $-112 \pm 57$ & $123 \pm 93$ & $-82 \pm 117$ & 0.757 \\
54181.05125 & 3724.7 & $79 \pm 50$ & $-53 \pm 82$ & $-46 \pm 105$ & 0.077 \\
54181.05320 & 3892.7 & $-159 \pm 65$ & $-462 \pm 98$ & $-394 \pm 125$ & 0.396 \\
54181.05514 & 4060.9 & $-114 \pm 57$ & $-214 \pm 95$ & $-269 \pm 122$ & 0.716 \\
54181.05709 & 4228.7 & $-36 \pm 57$ & $38 \pm 100$ & $151 \pm 128$ & 0.034 \\
\hline
\end{tabular}

Table 6. Individual measurements of the mean longitudinal magnetic field for the strongly magnetic star HD 94660.

\begin{tabular}{c|c|c}
\hline \hline MJD & $\left\langle B_{z}\right\rangle \pm \sigma_{B}$ & $\left\langle B_{z}\right\rangle \pm \sigma_{B}$ \\
& {$[\mathrm{G}]$} & {$[\mathrm{G}]$} \\
& set 1 & set 2 \\
\hline 54181.03960 & $-1534 \pm 30$ & $-1919 \pm 56$ \\
54181.04154 & $-1624 \pm 32$ & $-1861 \pm 60$ \\
54181.04348 & $-1688 \pm 34$ & $-1889 \pm 62$ \\
54181.04542 & $-1596 \pm 33$ & $-1908 \pm 62$ \\
\hline
\end{tabular}

that varies about a mean value of $\sim-1900 \mathrm{G}$ with a low amplitude ( $\sim 160 \mathrm{G}$ peak-to-peak) over a period of $2800 \mathrm{~d}$ (Mathys et al., in preparation).

The individual measurements of the longitudinal magnetic field obtained in 2004 and 2007 are presented in Table 5. The columns are, in order, the modified Julian date of the middle of the exposures, the time elapsed from the start of the first exposure, the mean longitudinal magnetic field $\left\langle B_{z}\right\rangle$ measured on Balmer lines and metal lines (set 1), the mean longitudinal magnetic field measurements restricted to the wavelength regions containing hydrogen Balmer lines (set 2), and the field measured on Balmer lines with the exception of the $\mathrm{H} \beta$ line (set 3 ). The last column gives the corresponding phase assuming a variability period of about $8.8 \mathrm{~min}$. The measurements for the strongly magnetic standard star HD 94660 are presented in Table 6.

Our observations obtained in 2007 March confirm the previous finding of the magnetic field variations on a short time scale. The observed magnetic field changes several times from positive

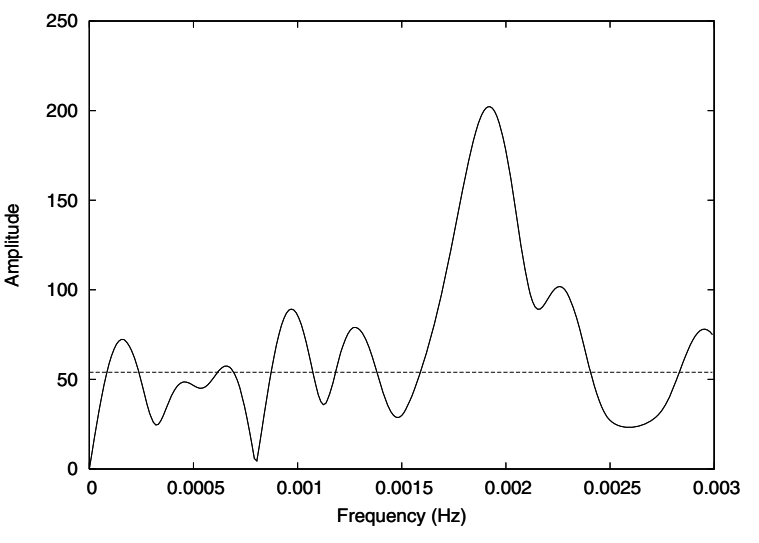

Fig. 6. Fourier spectrum for the magnetic field data derived from hydrogen lines without $\mathrm{H} \beta$. The horizontal line gives the noise level.

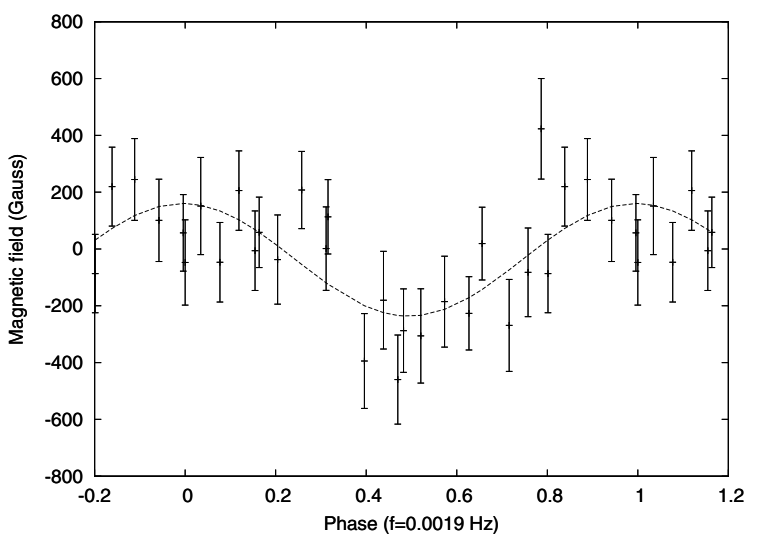

Fig. 7. Phase diagram for the same dataset as in Fig. 6 for the best candidate frequency of $0.0019 \mathrm{~Hz}$, i.e. a period of about $8.8 \mathrm{~min}$.

to negative values over the observing time span. However, only a few measurements are of the order of a few hundred Gauss at a significance level of more than $3 \sigma$. On the other hand, the amplitude of the variability of the magnetic field of our strongly magnetic standard star HD 94660 accounts only for $\sim 77$ G. To find a potential period of variations of the magnetic field in $\theta$ Car we performed a Fourier analysis of all three sets of magnetic field determinations. For the Fourier analysis we used Period04 (Lenz $\&$ Breger 2005) and searched for the best candidate frequency in the range $\left[0, f_{\mathrm{Nyq}}\right] \mathrm{Hz}$, where $f_{\mathrm{Nyq}}$ is the Nyquist frequency of $0.003 \mathrm{~Hz}$. As a result, we find that the frequency $0.0019 \mathrm{~Hz}$ is present in all three sets but with the highest $S / N$ level in set 3. For set 1 (with measurements of Balmer and metal lines) and for set 3 (with the $\mathrm{H} \beta$ line excluded), the highest peaks are at about $0.0019 \mathrm{~Hz}$, at a $3.0 \sigma$ level and $3.8 \sigma$ level, respectively. For set 2 , which includes the measurements on all available Balmer lines, only the second highest peak is at the same frequency, $0.0019 \mathrm{~Hz}$, at a $2.5 \sigma$ level. The Fourier spectrum for set 3 is shown in Fig. 6 with an amplitude of the magnetic field variation of $202 \pm 43 \mathrm{G}$. The corresponding phase diagram is shown in Fig. 7.

\section{Discussion}

\subsection{Binarity and evolutionary history}

We have presented in Sect. 2 the first high quality radial velocity curve for $\theta$ Car. It is well fitted by a Keplerian orbit with eccentricity $e=0.127$, leaving no doubt about the binary origin of 


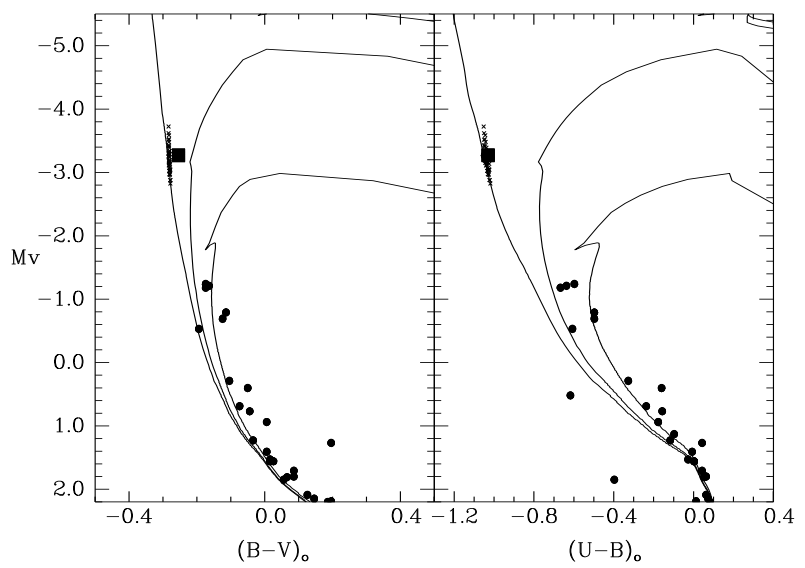

Fig. 8. Color-Magnitude diagram of IC 2602. Small crosses mark the position of main sequence stars with $T_{\text {eff }}=30000-32000 \mathrm{~K}$ and $\log g=4.0-4.4$, according to theoretical models. Solid lines represent isochrones for $\log \tau=3.0,7.5$, and 8.0. The photometric position of $\theta$ Car is marked with a filled square and other IC 2602 members with filled circles. Photometric data and cluster parameters $[(m-M)=6.1$, $E(B-V)=0.024]$ were taken from the WEBDA database (Mermilliod 1988).

these variations. The improved determination of the orbital parameters allows us to make a more sophisticated interpretation of the nature of this system and to review the possibility of a mass transfer between primary and secondary as the cause of the observed chemical anomalies. The physical parameters of $\theta$ Car determined in Sect. 3 suggest that it is located in the H-R diagram close to the ZAMS. According to the Geneva stellar models (Schaller et al. 1992; Lejeune \& Schaerer 2001) for solar abundances, the observed values of $T_{\text {eff }}=31000 \mathrm{~K}$ and $\log g=$ 4.2 correspond to a star with mass $M_{1}=15.25 M_{\odot}$, radius $R_{1}=$ $5.1 R_{\odot}$, and age $\log \tau=6.0$. Considering a $1 \sigma$ uncertainty in $\log g$ and $T_{\text {eff }}$, we obtain $\log \tau \leq 6.7$. This age value is considerably lower than the accepted age for the cluster IC $2602(\log \tau=7.83$, Kharchenko et al. 2005; $\log \tau=7.63$, Eggen 1972; $\log \tau=7.32$, Whiteoak 1961; $\log \tau=7.16$, Hill \& Perry 1969), consistent with the classification of this star as a blue straggler. We note that the absolute magnitude and intrinsic colors interpolated in the same theoretical grid are in agreement with the position of $\theta$ Car in the Color-Magnitude diagram of the cluster IC 2602 (see Fig. 8).

The uncertainty in the stellar mass and radius interpolated in the stellar model grid was estimated from the adopted errors for $T_{\text {eff }}$ and $\log g$. The primary mass ranges from $13.9 M_{\odot}$ (corresponding to a ZAMS star with $T_{\text {eff }}=30000 \mathrm{~K}$ ) to $18.2 M_{\odot}$ (corresponding to $T_{\text {eff }}=32000 \mathrm{~K}$ and $\log g=4.0$ ). From the estimated primary mass, relevant information for the system can be derived from the radial velocity curve, even when the orbital inclination is in principle unknown. We show in Fig. 9 the absolute value of the orbital semiaxis and the mass of the secondary star as a function of the orbital inclination. For each parameter plotted in this figure the solid line corresponds to the solution with $M_{1}=15.25 M_{\odot}$, while solutions with $13.9 M_{\odot}$ and $18.2 M_{\odot}$ are plotted with dotted lines.

To evaluate the occurrence of a mass exchange in the system, we calculated the volume radius of the Roche lobe at periastron $\left(R_{1}^{\text {crit }}\right.$ and $\left.R_{2}^{\text {crit }}\right)$, which are plotted in Fig. 9. From this figure it is clear that these parameters are not strongly dependent on the orbital inclination unless the latter is low. However, a low inclination can be ruled out for the following reasons. On the one hand, as argued by Walborn (1979), the absence of

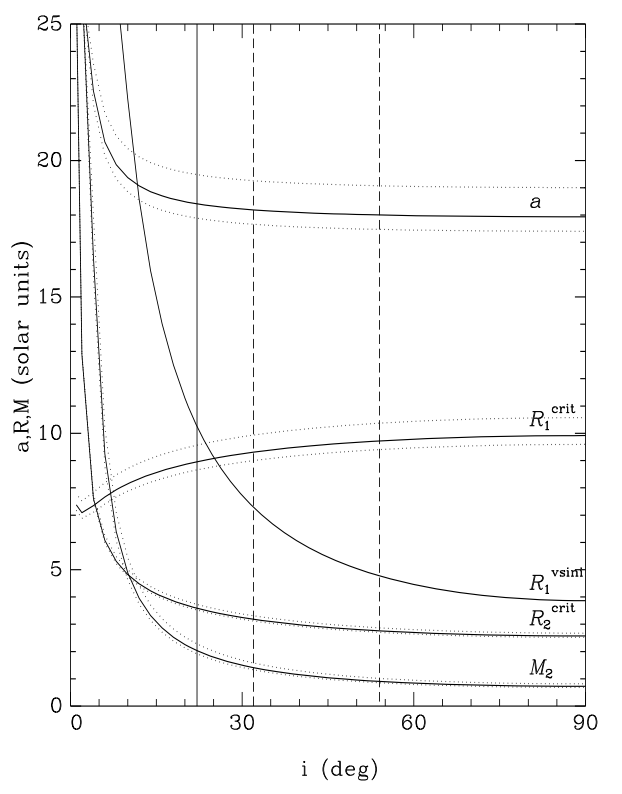

Fig. 9. Physical parameters as a function of the orbital inclination: orbital semiaxis $a$, critical radius for the primary $R_{1}^{\text {crit }}$ and the secondary $R_{2}^{\text {crit }}$, primary radius derived from the rotational velocity $R_{1}^{v \sin i}$, and mass of the secondary star $M_{2}$. The full lines correspond to $M_{1}=$ $15.25 M_{\odot}$ and the dotted lines to $M_{1}=13.9 M_{\odot}$ and $M_{1}=18.2 M_{\odot}$.

a pronounced emission at $\mathrm{H} \alpha$ indicates that the equatorial velocity is lower than $300 \mathrm{~km} \mathrm{~s}^{-1}$ and consequently, from the observed $v \sin i$ value we obtain $i \geq 22^{\circ}$. This value is presented by the solid vertical line in Fig. 9 and corresponds to an upper limit of about $2.0 M_{\odot}$ for the secondary component. On the other hand, if we assume that the rotation is synchronized with the orbital motion at periastron, the relatively small radius deduced from the spectroscopic value of $\log g$ is compatible with the observed $v \sin i$ only for high inclinations. For the case of synchronous rotation we derive a rotational period of $1.692 \pm$ $0.007 \mathrm{~d}$ and hence $R_{1} \sin i=3.78 \pm 0.27 R_{\odot}$. The adopted range for $M_{1}\left(13.9-18.2 M_{\odot}\right)$ corresponds to $R_{1}=4.7-7.1 R_{\odot}$, and therefore we obtain $i=32^{\circ}-54^{\circ}$. These limits are marked with two dashed vertical lines in Fig. 9. Interestingly, this rotational period of $1.692 \pm 0.007 \mathrm{~d}$ is in good agreement with the $1.779 \mathrm{~d}$ period discovered by Walborn (1979) who studied intensity variations of Si IV $\lambda 4089$.

The radius derived from $v \sin i$ assuming synchronization would coincide with the critical radius for $i \approx 25^{\circ}$. In this case $R_{1} \approx 9.1 R_{\odot}$ and $\log g \approx 3.7$, which is lower than the spectroscopic value by $2.5 \%$. Therefore, we expect that, at present, the primary star is probably not filling its Roche lobe, although it is close to that point.

A star of $15.25 M_{\odot}\left(13.9-18.5 M_{\odot}\right)$ with a current radius of $5.1 R_{\odot}\left(4.7-7.1 R_{\odot}\right)$ has an evolutionary age of $1.0 \mathrm{Myr}$ (0-4.3 Myr) and, evolving as a single star, would have at the Terminal Age Main Sequence (TAMS) a radius of about $13.6 R_{\odot}$ $\left(12.6-16.3 R_{\odot}\right)$, which is significantly larger than the critical radius. Thus, we can expect mass-transfer to occur from the primary to the secondary during the main-sequence stage, at an evolutionary age of about $11 \mathrm{Myr}$.

Since $\theta$ Car is a bona-fide blue straggler of IC 2602, it is very likely that the system has suffered in the past a mass-transfer in the opposite direction. Eggen \& Iben (1988) have discussed extensively this possibility as an explanation for its abnormal position in the Color-Magnitude diagram. The apparent evolutionary youth of $\theta$ Car and its peculiar chemical abundances are best 
understood if the present primary star was originally the lessmassive component and has accreted mass transfered from the companion. Its original mass could be less than half of the total mass of the system, similar to other stars near the turnoff point in the cluster. After considerable mass-transfer, it would appear as a non-evolved massive star above the cluster turnoff and near the ZAMS. As was noted by Eggen \& Iben (1988), during the evolution of this binary system an important loss of angular momentum has taken place, making it possible to achieve a system with such a short period and low mass-ratio. The orbital angular momentum of a binary system can be written as

$J_{\text {orb }}=\frac{2 \pi}{P} M a^{2} \frac{q}{(1+q)^{2}} \sqrt{1-e^{2}}$

while for the rotational momentum of each star we have

$J_{\text {rot }}=\frac{2 \pi}{P_{\text {rot }}} \beta^{2} M R^{2}$

where $\beta$ is the radius of gyration, which is typically 0.25 for a main-sequence star (Claret \& Giménez 1989). In any case the rotational contribution to the total angular momentum is small. We can use these expressions to calculate the angular momentum of the system and compare the present value with that at the moment when the mass-ratio was close to unity. As illustration, let us assume that the original primary star (presently the secondary) with $9 M_{\odot}$ filled its Roche lobe when it was still in the main-sequence with a radius of the order of 7-9 $R_{\odot}$. Therefore, at that time the separation between the stars was about $20-25 R_{\odot}$. Assuming a conservative mass transfer and adopting for the present configuration $M_{1}=15 M_{\odot}, M_{2}=1.0 M_{\odot}$, and $a=19 R_{\odot}$, we can estimate that the present angular momentum is about $0.22-0.25$ of the original value. In conclusion, most of the angular momentum has been lost.

\subsection{Abundances and magnetic field}

The binary and blue straggler nature of $\theta$ Car leads us to relate the observed chemical anomalies to the past history of the binary system. First, mass transfer of chemically processed material from the initially more massive component has dramatically altered the CNO surface abundances. Second, the spun up phase that ensued may have further enhanced nitrogen and depleted carbon in $\theta$ Car as a result of rotational mixing (see e.g. Langer et al. 2008). The tendency of magnetic B stars to show a higher incidence of an $\mathrm{N}$ excess compared to stars with no field detection (Morel et al. 2008) also indicates that magnetic phenomena could play a role. The results of the previous studies, along with the fact that little is known about the magnetic properties of hot, massive stars, has motivated our search for a magnetic field in $\theta$ Car.

There are only six massive stars with detected magnetic fields that are hotter than $\theta$ Car: $\theta^{1}$ Ori C, HD 191612, HD 155806, HD 148937, 9 Sgr, and $\tau$ Sco. The star HD 191612 with a rotation period of $538 \mathrm{~d}$ was observed with ESPaDOnS only during four consecutive nights (Donati et al. 2006a). Among the published magnetic field measurements of $\theta^{1}$ Ori C, only four measurements show significance at the $3 \sigma$ level (Wade et al. 2006). HD 155806 and HD 148937 were observed only once and 9 Sgr three times (Hubrig et al. 2007; Hubrig et al., in preparation). The sixth star, $\tau$ Sco, with physical parameters very similar to those of $\theta$ Car, was studied over the rotational period of $41 \mathrm{~d}$. It possesses the weakest mean longitudinal magnetic field with a maximum value of $88 \mathrm{G}$ and a very complicated geometry. The structure of its magnetic field topology features in particular a significantly warped torus of closed magnetic loops encircling the star and additional smaller networks of closed field lines (Donati et al. 2006b).

In our magnetic field study of $\theta$ Car, only very few measurements were achieved at a significance level of $3 \sigma$ and their sporadic appearance is difficult to explain in the framework of the presence of a global large-scale organized magnetic field. On the other hand, the presence of a complex structure of the magnetic field in $\theta$ Car may hypothetically be possible in an analogous manner to the magnetic field topology of $\tau$ Sco. The detected periodicity of the order of $8.8 \mathrm{~min}$ in the dataset of measurements carried out on hydrogen Balmer lines with the exclusion of $\mathrm{H} \beta$ is surprising, and if it is not spurious, its discovery would give rise to the important question whether the presence of pulsations could cause such a periodicity. No studies of short-time pulsations exist for $\theta$ Car so far. B0 main-sequence stars are expected to pulsate in low radial order $\mathrm{p}$ - and g-modes with periods of the order of hours (typically 3 to $8 \mathrm{~h}$ ). For such massive stars, periods of the order of a few minutes would correspond to high radial order p-modes, but non-adiabatic codes do not predict their excitation. However, since current models do not take into account the presence of a magnetic field, one cannot exclude the possibility that magnetic fields would favour the excitation of these types of modes in analogy to roAp stars which do possess a magnetic field and pulsate in high radial order modes of the order of a few minutes. Our data with the CORALIE spectrograph were taken typically every $7 \mathrm{~min}$. This leads to a sampling rate which is too low to allow us to detect variations of spectral line profiles on short time scales.

Since the mechanism of the generation and the maintenance of magnetic fields in massive stars is not well understood yet, we are cautious in drawing any conclusion on the presence and the behaviour of a magnetic field in $\theta$ Car. Only additional timeresolved magnetic field observations will tell us about the presence and the structure of the magnetic field geometry of $\theta$ Car and will help to discriminate among the hypotheses described above.

Acknowledgements. T.M. acknowledges financial support from the Research Council of Leuven University through grant GOA/2003/04. We are very grateful to C. Aerts and M. Desmet for acquiring the CORALIE spectra of $\tau$ Sco, to V. Elkin and P. North for the FEROS spectra of $\theta$ Car, and to M. E. Veramendi for acquiring the CASLEO spectra. We also wish to thank L. Decin and M. F. Nieva for their help on the construction of the model grid at high temperatures, K. Lefever for communicating her results prior to publication, and Y. Nazé and the referee P. Dufton for valuable comments.

\section{References}

Borra, E. F., \& Landstreet, J. D. 1979, ApJ, 228, 809

Cohen, D. H., de Messières, G. E., MacFarlane, J. J., et al. 2003, ApJ, 586, 495 Claret, A., \& Giménez, A. 1989, A\&AS, 81, 37

Daflon, S., \& Cunha, K. 2004, ApJ, 617, 1115

Donati, J.-F., Howarth, I. D., Bouret, J.-C., et al. 2006a, MNRAS, 365, 6

Donati, J.-F., Howarth, I. D., Jardine, M. M., et al. 2006b, MNRAS, 370, 629

Eggen, O. J. 1972, ApJ, 173, 63

Eggen, O. J., \& Iben, I. 1988, AJ, 96, 635

Gagné, M., Oksala, M. E., Cohen, D. H., et al. 2005, ApJ, 628, 986

Gummersbach, C. A., Kaufer, A., Schäfer, D. R., et al. 1998, A\&A, 338, 881

Henrichs, H. F., Schnerr, R. S., \& Ten Kulve, E. 2005, in The Nature and Evolution of Disks Around Hot Stars, ed. R. Ignace, \& K. G. Gayley, ASP Conf. Ser., 337, 114

Hill, G., \& Perry, C. L. 1969, AJ, 74, 1011

Hubrig, S., Briquet, M., Schöller, M., et al. 2006, MNRAS, 369, 61

Hubrig, S., North, P., \& Schöller, M. 2007, Astron. Nachr., 328, 475

Kharchenko, N. V., Piskunov, A. E., Roeser, S., et al. 2005, A\&A, 438, 1163

Kilian, J. 1994, A\&A, 282, 867

Kilian-Montenbruck, J., Gehren, T., \& Nissen, P. E. 1994, A\&A, 291, 757 
Kudritzki, R. P. 1976, A\&A, 52, 11

Kurucz, R. L. 1993, ATLAS9 Stellar Atmosphere Programs and $2 \mathrm{~km} \mathrm{~s}^{-1}$ grid. Kurucz CD-ROM, 13 (Cambridge, Mass.: Smithsonian Astrophysical Observatory)

Kurucz, R. L., \& Bell, B. 1995, Atomic Line Data, ed. R. L. Kurucz, \& B. Bell, Kurucz CD-ROM, 23 (Cambridge, Mass.: Smithsonian Astrophysical Observatory)

Langer, N., Cantiello, M., Yoon, S. -C., et al. 2008, in Proc. IAU-Symp., 250, on Massive Stars as Cosmic Engines, ed. F. Bresolin, P. Crowther, \& J. Puls, in press [arXiv:0803.0621]

Lefever, K. 2007, Ph.D. Thesis, Katholieke Universiteit Leuven, Belgium

Lejeune, T., \& Schaerer, D. 2001, A\&A, 366, 538

Lenz, P., \& Breger, M. 2005, CoAst, 146, 53

Levato, H., Morrell, N., Garcia, B., \& Malaroda, S. 1988, ApJS, 68, 319

Lloyd, C., Stickland, D. J., \& Walborn, N. R. 1995, PASP, 107, 1030

Lyubimkov, L. S., Rostopchin, S. I., \& Lambert, D. L. 2004, MNRAS, 351, 745

Maeder, A. 1987, A\&A, 178, 159

Mermilliod, J. C. 1988, Bulletin d'Information du Centre de Donnees Stellaires, 35,77

Meynet, G., \& Maeder, A. 2003, A\&A, 404, 975

Mokiem, M. R., de Koter, A., Puls, J., et al. 2005, A\&A, 441, 711

Morel, T., Butler, K., Aerts, C., et al. 2006, A\&A, 457, 651

Morel, T., Hubrig, S., \& Briquet, M. 2008, A\&A, 481, 453

Nazé, Y., Rauw, G., Pollock, A. M. T., et al. 2007, MNRAS, 375, 145
Nieva, M. F., \& Przybilla, N. 2007, A\&A, 467, 295

Puls, J., Urbaneja, M. A., Venero, R., et al. 2005, A\&A, 435, 669

Randich, S., Schmitt, J. H. M. M., Prosser, C. F., \& Stauffer, J. R. 1995, A\&A, 300, 134

Rauw, G., Morrison, N. D., Vreux, J.-M., et al. 2001, A\&A, 366, 585

Repolust, T., Puls, J., Hanson, M. M., et al. 2005, A\&A, 440, 261

Saesen, S., Briquet, M., \& Aerts, C. 2006, CoAst, 147, 109

Schaller, G., Schaerer, D., Meynet, G., \& Maeder, A. 1992, A\&AS, 96, 269

Schild, H., \& Berthet, S. 1986, A\&A, 162, 369

Schönberner, D., Herrero, A., Becker, S., et al. 1988, A\&A, 197, 209

Simón-Díaz, S., Herrero, A., Esteban, C., \& Najarro, F. 2006, A\&A, 448, 351

Trundle, C., Dufton, P. L., Hunter, I., et al. 2007, A\&A, 471, 625

Vanbeveren, D., \& de Loore, C. 1994, A\&A, 290, 129

Wade, G. A., Fullerton, A. W., \& Donati, J.-F. 2006, A\&A, 451, 195

Walborn, N. R. 1976, ApJ, 205, 419

Walborn, N. R. 1979, PASP, 91, 442

Walborn, N. R. 2006, in The Ultraviolet Universe: Stars from Birth to Death, 26th meeting of the IAU, Joint Discussion 4, 16-17 August, Prague, Czech Republic, JD04, \#19

Walker, H. J., \& Hill, P. W. 1985, A\&AS, 61, 303

Whiteoak, J. B. 1961, MNRAS, 123, 245

Wilson, R. E., \& Sanford, R. E. 1915, Lick Obs. Bull., 8, 124

Woosley, S. E., \& Heger, A. 2006, ApJ, 637, 914 